\title{
Analysis of microRNA profile of Anopheles sinensis by deep sequencing and bioinformatic approaches
}

\author{
Xinyu Feng ${ }^{1,2}$, Xiaojian Zhou ${ }^{3}$, Shuisen Zhou ${ }^{1 *}$, Jingwen Wang ${ }^{4^{*}}$ and Wei $\mathrm{Hu}^{1,4^{*}}$
}

\begin{abstract}
Background: microRNAs (miRNAs) are small non-coding RNAs widely identified in many mosquitoes. They are reported to play important roles in development, differentiation and innate immunity. However, miRNAs in Anopheles sinensis, one of the Chinese malaria mosquitoes, remain largely unknown.

Methods: We investigated the global miRNA expression profile of An. sinensis using Illumina Hiseq 2000 sequencing. Meanwhile, we applied a bioinformatic approach to identify potential miRNAs in An. sinensis. The identified miRNA profiles were compared and analyzed by two approaches. The selected miRNAs from the sequencing result and the bioinformatic approach were confirmed with qRT-PCR. Moreover, target prediction, GO annotation and pathway analysis were carried out to understand the role of miRNAs in An. sinensis.

Results: We identified 49 conserved miRNAs and 12 novel miRNAs by next-generation high-throughput sequencing technology. In contrast, 43 miRNAs were predicted by the bioinformatic approach, of which two were assigned as novel. Comparative analysis of miRNA profiles by two approaches showed that 21 miRNAs were shared between them. Twelve novel miRNAs did not match any known miRNAs of any organism, indicating that they are possibly species-specific. Forty miRNAs were found in many mosquito species, indicating that these miRNAs are evolutionally conserved and may have critical roles in the process of life. Both the selected known and novel miRNAs (asi-miR-281, asi-miR-184, asi-miR-14, asimiR-nov5, asi-miR-nov4, asi-miR-9383, and asi-miR-2a) could be detected by quantitative real-time PCR (qRT-PCR) in the sequenced sample, and the expression patterns of these miRNAs measured by qRT-PCR were in concordance with the original miRNA sequencing data. The predicted targets for the known and the novel miRNAs covered many important biological roles and pathways indicating the diversity of miRNA functions. We also found 21 conserved miRNAs and eight counterparts of target immune pathway genes in An. sinensis based on the analysis of An. gambiae.
\end{abstract}

Conclusions: Our results provide the first lead to the elucidation of the miRNA profile in An. sinensis. Unveiling the roles of mosquito miRNAs will undoubtedly lead to a better understanding of mosquito biology and mosquito-pathogen interactions. This work lays the foundation for the further functional study of An. sinensis miRNAs and will facilitate their application in vector control.

Keywords: microRNA, Next-generation sequencing, Bioinformatic approach, Anopheles sinensis

\footnotetext{
*Correspondence: zss163@hotmail.com; jingwenwang@fudan.edu.cn;

huwwyz@163.com

${ }^{1}$ National Institute of Parasitic Diseases, Chinese Center for Disease Control and Prevention, Key Laboratory of Parasite and Vector Biology, National Health and Family Planning Commission, WHO Collaborating Center for Tropical Diseases, National Center for International Research on Tropical Diseases, Shanghai 200025, People's Republic of China

${ }^{4}$ State Key Laboratory of Genetic Engineering, Ministry of Education Key Laboratory of Contemporary Anthropology, Collaborative Innovation Center for Genetics and Development, School of Life Sciences, Fudan University, Shanghai 200438, People's Republic of China

Full list of author information is available at the end of the article
} 


\section{Background}

Malaria is a life-threatening infectious disease caused by the Plasmodium spp. parasites and transmitted to individuals through the bites of infected Anopheles mosquitoes. Although the global epidemiology of malaria has changed dramatically, still 198 million malaria cases occurred, leading to 584,000 deaths in 2013 [1]. In China, a total number of 3078 malaria cases were reported in 2014, among them, vivax malaria accounting for $89.29 \%$ of the indigenous malaria cases [2]. Currently, China is implementing a National Malaria Elimination Program. However, indigenous malaria in some places, together with the great challenge posed by imported Plasmodium falciparum malaria [3], would definitely hinder the process of elimination in the coming years.

Anopheles sinensis, one of the important malaria vectors in China, is still considered as the primary vector of $P$. vivax malaria due to its wide distribution and high density. Anopheles sinensis is a facultative mosquito species, which can take blood meals both from humans and animals but is preferably zoophilic according to a previous ecological study [4]. Malaria used to be prevalent in most parts of the flatlands in China between 25 and $33^{\circ}$ $\mathrm{N}$ where this species was incriminated as the most dominant malaria vector [5]. According to the recent vector surveillance data, the dominant status of the mosquito has not undergone any fundamental changes, although the natural breeding site environment has changed significantly [6]. To make matters worse, a globally warmer climate [4] and the emerging resistance to a variety of insecticides [7] would together blur the prospect of malaria elimination in China. Effective integrated vector control research is needed to sustain the gains achieved so far and to achieve global malaria elimination in the future.

miRNAs are single-stranded and small non-coding RNAs that play significant roles in the control of gene expression by regulating the expression of target genes at a post-transcriptional level [8]. Through their complicated gene regulatory networks with other non-coding RNAs [9], miRNAs have important functions in most fundamental biological processes including apoptosis, proliferation, differentiation, development, cell cycle control and metabolism [10]. Since the discovery of the first miRNA in Caenorhabditis elegans in 1994 by the joint efforts of Victor Ambros's and Gary Ruvkun's laboratories $[11,12]$, thousands of miRNAs have been identified in various organisms, such as mammals, nematodes, and plants, insects and even viruses which were generally believed could not encode miRNAs due to nucleus inaccessibility in the past.

Recently, the miRNA profiles of several mosquito species (Anopheles gambiae, Anopheles stephensi, Aedes aegypti, Culex fatigans and Aedes albopictus among others) have been successfully identified and characterized [13-16]. Several studies suggested that miRNAs are dysregulated and could play important roles at the post-transcriptional level in physiology, pathogenesis, and immunology. Some of the miRNAs have been frequently demonstrated to be functionally involved in the blood meal, parasite infection or virus infection, such as members of the miR-275 [17], miR-305 [18, 19] and miR-34 [20], indicating that these dysregulated miRNAs may play pivotal roles in the interplay between host and pathogen. However, although $A n$. sinensis is an important vector in China, its miRNAs profile and the roles of miRNAs remain largely unfulfilled.

In this study, we performed a comprehensive profiling of miRNAs by high throughput small RNA sequencing and an alternative bioinformatic approach to investigate the miRNAs in An. sinensis. Comparisons were made between two approaches to assess the discrimination and difference of identified miRNA profiles. In addition, candidate miRNAs (both conserved and novel miRNAs) validated by two methods were subject to miRNA-gene network analysis to predict the targets of these miRNAs. Furthermore, we investigate the potential roles of miRNAs in An. sinensis.

\section{Methods}

\section{Mosquito rearing and sample preparation}

Anopheles sinensis (China strain) mosquitoes were reared under insectary in screened cages at $28 \pm 2{ }^{\circ} \mathrm{C}$, $70-75 \%$ humidity. Adult mosquitoes were provided with constant access to sterile glucose solution and water soaked sponges. After eclosion, adult female samples were pooled with $250 \mu \mathrm{l}$ of $\mathrm{TRIzol}^{\circledR}$ reagent (Invitrogen, California, USA) immediately following collection and stored at $-80{ }^{\circ} \mathrm{C}$ until further processing. Total RNA was extracted from samples for miRNAs (Takara, Dalian, China) followed manufacturer's protocol. Quality and purity of RNA were assessed by electrophoresis and ultraviolet spectrophotometry, together with the ratio of OD260 and OD280, of which the value of all samples ranged from 1.8 to 2.2. RNA integrity assess used the Agilent2100 bioanalyzer (Agilent Technologies, Santa Clara, CA, USA). The RIN (RNA integrity number) value of all samples ranged from 8.1-8.9 (scale 1-10), indicating high-quality RNA.

\section{Small RNA library construction and Illumina sequencing} Small RNAs (sRNAs) libraries were made as manufacturer's instructions (Illumina Inc, San Diego, USA). sRNAs were isolated by separating total RNAs on denaturing polyacrylamide gel electrophoresis and cutting a portion of the gel corresponding to the size 18-30 nucleotides. The RNAs were ligated with $3^{\prime}$ and 5' RNA adaptors. The adaptor-ligated sRNAs were subjected to RT-PCR with 15 cycles of PCR amplification. After gel 
purification of amplified cDNAs, approximately $20 \mu \mathrm{g}$ of the pooled RNA samples were submitted for sequencing (Shanghai OE Biotech Co., Shanghai, China) using the Illumina HiSeq 2000.

\section{Analysis of small RNA sequencing data}

First, the initial reads obtained from Illumina sequencing were pretreated to remove adaptors, poly A reads, reads without the insert fragment, and reads of less than $10 \mathrm{nt}$. Next, the remaining sequences (clean reads) were mapped to the An. sinensis genome (GenBank assembly accession: GCA_000441895.2) using SOAP [21] with a tolerance of one mismatch to analyze their distribution. The matched sequences were then queried against GenBank miRBase [22], Rfam [23], and repeats database [24] to annotate the sRNA (rRNAs, tRNAs, snRNAs, snoRNA, miRNA) sequences. Reads overlapping with the exons of proteincoding genes were also excluded to avoid mRNAs contamination. The remnant reads were compared to the miRBase database to identify the conserved miRNAs. Filtered unmatched clean reads were used for miRNA prediction to identify novel miRNAs. The novel miRNAs candidates were predicted by MiRDeep [25] only if they met the criteria described by Allen et al. [26] and Friedlander et al. [27]. The secondary structure, the Dicer cleavage site and the minimum free energy of the unannotated small RNA reads, which could be mapped to An. sinensis genome sequences were predicted by using RNA-fold (http://rna.tbi.univie.ac.at/).

\section{Bioinformatic approach for identification of An. sinensis miRNA}

We used transcriptome analysis method to identify miRNAs from An. sinensis. A total of 3119 stem-loop premiRNA sequences belonging to 26 organisms of the subphylum Hexapoda were extracted from miRBase v.21 [22]. RNAseq transcripts of An. sinensis deposited in Vectorbase were also downloaded. Pre-miRNAs were used as the query for the search of its homolog in $A n$. sinensis transcriptome by the BLAST2.2.22+ program at as described by Krishnan et al- [28] with all parameters as default. The FASTA formats of all the candidate sequences were screened and saved. Prediction and validation of the indentured secondary structures were performed using the M-Fold tool [29] based on thermodynamics metrics. The annotation of mature miRNA was performed based on the widely accepted criteria for selecting miRNAs from pre-miRNA sequences as previously described [30]. Nomenclature of predicted miRNAs was assigned as the pattern of miRBase and prefixed with "asi" to denote An. sinensis. The mature sequences were designated "miR" and the precursor hairpins were labeled as "mir".
miRNA expression validated by quantitative qRT-PCR

To validate the presence and expression of the identified miRNAs, eight miRNAs with differential expression were selected for qRT-PCR as described previously [31]. The total RNA was extracted using TRIzol Reagent (Invitrogen). The reverse transcription reaction was performed with RevertAid First Strand cDNA Synthesis Kit (Fermentas, Carlsbad, USA), and the reverse-transcribed products were used as the template for qRT-PCR with miRNA-specific primers (Additional file 1: Table S1). All reactions were assayed in three biological and technical replications and performed in an ABI-7300 (Applied Biosystems, Foster City, USA) using SYBR Green qPCR kit (Thermo Fisher, San Diego, USA). The relative expression level of miRNA was normalized to the internal control of U6 small nuclear (U6). PCR conditions consisted of pre-denaturation and hot start Taq activation at $95{ }^{\circ} \mathrm{C}$ for $10 \mathrm{~min}$, then 40 cycles of $95{ }^{\circ} \mathrm{C}$ for $15 \mathrm{~s}$, and $60{ }^{\circ} \mathrm{C}$ for $45 \mathrm{~s}$, and the relative expression was calculated by using the $2^{-\Delta \Delta \mathrm{Ct}}$ method.

\section{miRNA target gene prediction, GO enrichment and KEGG pathway analysis}

In the present study, the target genes of identified miRNAs were predicted using an in silico RNA-RNA hybridization approach following the protocol reported by Kruger \& Rehmsmeier [32]. The program includes features such as: (i) perfect miRNA seed complementarity (positions 2 to 8 ) with 3 'UTR sequence; (ii) $P$-value < 0.05; (iii) miRNA:mRNA binding energy $<-20 \mathrm{kcal} / \mathrm{mol}$; (iv) 1 maximum number of loops and internal bulges allowed; (v) no more than two adjacent mismatches in the miRNA/target duplex. 3'UTRs of An. gambiae downloaded from UTRdb [33] were used for the prediction. Mature miRNA sequence (fasta) and downloaded 3'UTR sequence was used as "query" and "target" files in RNAhybrid. The identities of predicted miRNA targets were determined by Kobas 3.0 [34] using An. gambiae as the reference species to limit annotations. Genes included in both the significant GO terms and KEGG pathways were further screened (enrichment score $>2$ and $P<0.05$ ). miRNA-mRNA network analysis was conducted for these selected targets and visualized by Cytoscape [35].

\section{Results}

\section{Overview of An. sinensis sRNAs by sequencing}

We obtained a total of 24,502,168 raw sequence reads from a constructed small RNA library. After removing the low quality, adaptor sequences, and junk sequences, $16,586,625$ high-quality sequence reads were retained for subsequent analysis (Table 1). Of these, $91.30 \%$ of the total reads and $85.55 \%$ of the unique reads mapped perfectly to the An. sinensis genome assembly. The length distributions of the total sRNA reads in the library are 
outlined in Fig. 1. The majority of sRNAs were between 19 and $24 \mathrm{nt}$, and the most abundant size class distribution was $22 \mathrm{nt}$, which accounted for $8.14 \%$ of the library, followed by $23 \mathrm{nt}$ (7.54\%), while the typical size range for Dicer-derived sRNA products are 20-24 nt. We removed a class of sRNAs about 24-27 nt in length (repeat-associated small interfering RNAs: rasiRNAs) which mediate the silencing of genomic repeats and transposons. We also removed sRNAs at 32-35 nt peak which represents longer piRNA-like small RNAs. The remaining reads were subsequently used for the identification of conserved miRNAs and the prediction of novel miRNAs.

\section{Identification of conserved miRNAs and novel miRNAs by deep sequencing}

To identify conserved miRNAs in An. sinensis, the remaining small RNA sequences were Blastn searched against miRBase v.21 [22]. A total of 73 miRNAs were identified in An. sinensis. Among them, 49 miRNAs were identified as conserved (irrespective of $5 p$ and $3 p$ ), and these miRNAs belong to 46 different miRNA families (Additional file 2: Table S2). The top ten most abundant miRNAs are asi-miR-281, asi-miR-8, asi-miR-14, asi-miR277, asi-miR-276, asi-miR-1891, asi-miR-10, asi-miR-184, asi-miR-9c, asi-miR-316 and asi-miR-275. After identifying the conserved miRNAs described above, the unannotated sRNAs from the library were subjected to novel miRNAs prediction by searching against the An. sinensis genome. Moreover, we also considered the potential of miRNA precursors or sequences to form a hairpin structure. In total, 12 miRNA candidates were assigned as novel miRNAs as shown in Table 2. Novel miRNAs were tentatively classified into known miRNAs families by blasting against miRBase. However, they showed no conserved seed region with known miRNAs from other species. These miRNA sequences were designated in a specific order as detailed in Table 2.

Table 1 Categorization of reads of small RNAs in An. sinensis

\begin{tabular}{lll}
\hline Category & No. of reads & Percent (\%) \\
\hline Total reads & $24,502,168$ & 100 \\
Reads trimmed adaptor & 58,308 & 0.237970779 \\
Reads trimmed N & 41,501 & 0.169376849 \\
Reads trimmed Quality Dynamics & 11 & $4.4894 \mathrm{E}-05$ \\
Reads trimmed Quality Percent & 1387 & 0.005660724 \\
Reads trimmed PolyA/T & 9973 & 0.040702521 \\
Reads trimmed length & $7,804,363$ & 31.8517243 \\
Clean reads & $16,586,625$ & 67.69451993 \\
\hline
\end{tabular}

\section{Overview of An. sinensis miRNAs by the bioinformatic approach}

Our pipeline yielded a number of putative miRNAs by nucleotide BLAST of 3119 stem-loop reference sequences resulting in 68 orthologous pre-miRNA signatures in 26 searched organisms of the subphylum Hexapoda. After removing the homologues, 43 miRNAs were identified in the in silico search (Additional file 3: Table S3). Among them, we have noted that 41 miRNAs have been deposited in Vectorbase, so only two $A n$. sinensis miRNAs were reported as new after a careful evaluation of the secondary structure analysis (Fig. 2). These two miRNAs were noticed in plus strand and had the minimum free energy of -24.2 and $-29.5 \mathrm{kcal} / \mathrm{mol}$, respectively. Then, miRNAs were named according to the mirBase naming convention as asi-mir-9383 and asimir-2a-2 (as shown in Table 3). These two miRNAs identified in our study had homologues with mature miRNAs from insect species other than mosquitoes indicating that they may be species-specific.

\section{Validation of predicted miRNA by qRT-PCR}

To identify the presence and the relative expression of the predicted miRNA, we selected known miRNAs and novel miRNAs from both the deep sequencing and the bioinformatic approach to perform qRT-PCR (Fig. 3a). All miRNAs (asi-miR-281, asi-miR-184, asi-miR-14, asimiR-nov5, asi-miR-nov4, asi-miR-9383 and asi-miR-2a) showed in the sample. We also observed a similar trend for the expression patterns of these miRNAs measured by qRT-PCR when compared with that in the original miRNA profiling data (Fig. 3b), which in turn confirmed the sequencing result.

\section{Comparative analysis of miRNA profiles by the deep sequencing and the bioinformatic approach}

Analysis of the miRNAs profiles by the deep sequencing and the bioinformatic approach is shown in Fig. 4a. A total of 84 miRNAs were identified by combining two approaches, among them, 21 miRNAs were shared between them. As for the 14 novel miRNAs in this study, two miRNAs were previously reported for Drosophila melanogaster and 12 did not match any known miRNAs in any organism. Compared with miRNA genes predicted from the genome of An. sinensis by Dritsou et al. [36] (Additional file 4: Table S4), 19 miRNAs (asi-miR-252, asimiR-10, asi-miR-927, asi-miR-8, asi-miR-996, asi-miR-1, asi-miR-276, asi-miR-305, asi-miR-277, asi-let-7, asi-miR283, asi-miR-210, asi-miR-133, asi-miR-988, asi-miR-275, asi-miR-315, asi-miR-14, asi-miR-31 and asi-miR-999) in the An. sinensis were shared within three databases as shown in Fig. 4b. Moreover, we also compared the miRNA profile of An. sinensis with miRNAs for An. gambiae, Ae. aegypti and $C x$ quinquefasciatus. The result showed that 


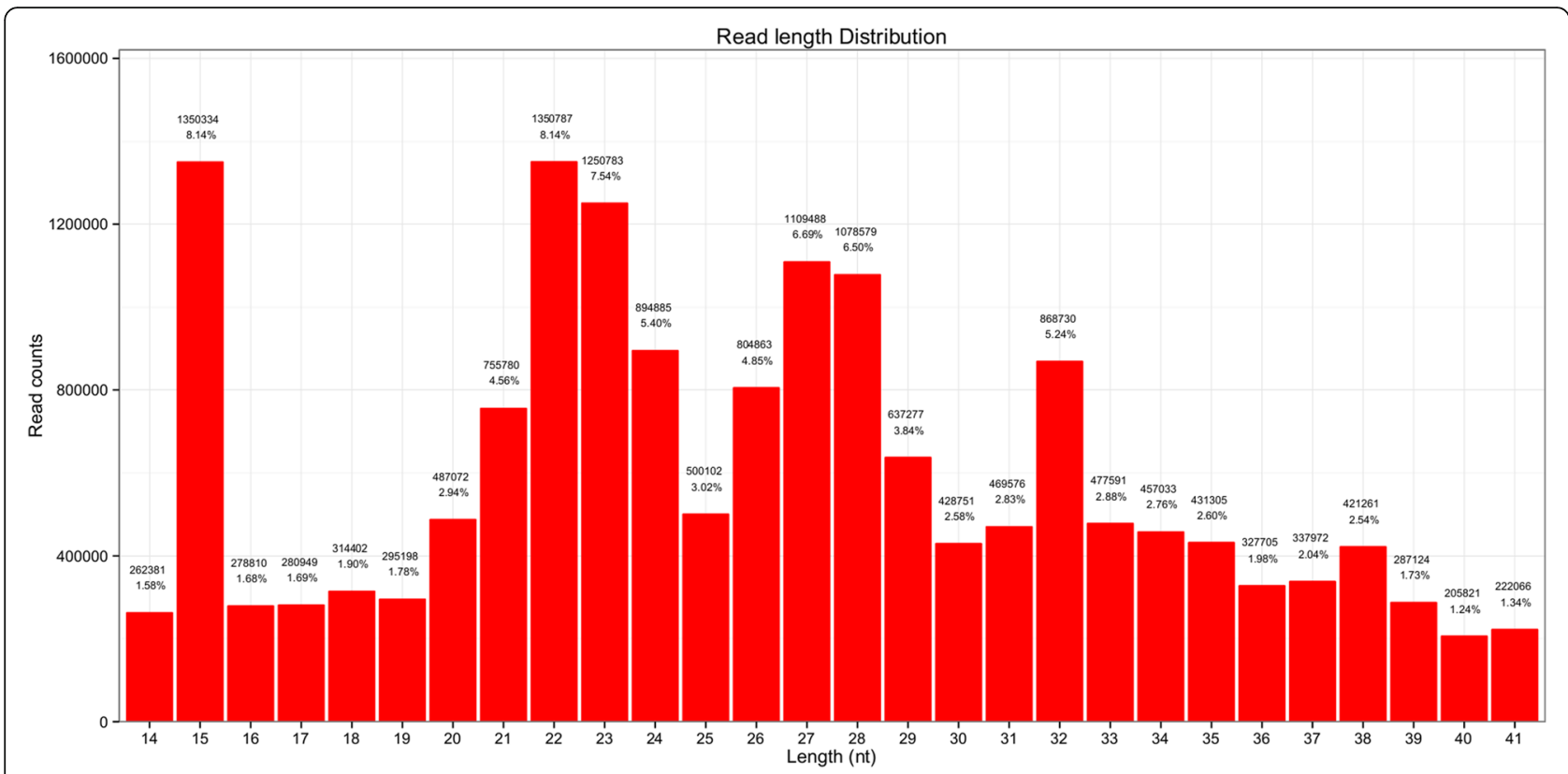

Fig. 1 Length distribution for total sRNA reads of the An. sinensis library

there is a high consistency ( 40 miRNAs) as far as total numbers of miRNAs identified in these taxa as shown in Fig. 4c.

\section{Novel miRNA target gene prediction, GO enrichment and KEGG pathway analysis}

The putative targets for the 14 novel miRNAs were performed using RNAHybrid to understand the putative biological roles. A total of 938 potential targets that satisfied the preset values were predicted (Additional file 5: Table S5). Maximum and minimum numbers of mRNA targets were predicted for asi-miR-nov11 $(n=173)$ and asi-miR-nov1/asi-miR-2a-2 $(n=20)$, respectively. The targets were further analyzed by KOBAS. The predicted targets were found to be involved in a broad range of biological processes. The top 10 significant GO terms and KEGG pathways for the 14 novel miRNAs and putative target genes, respectively, are shown in Fig. 5. The most significantly enriched $\mathrm{GO}$ terms included ribosome (GO: 0005840), neutral lipid metabolic process (GO: 000 6638), acylglycerol metabolic process (GO: 0006639), triglyceride metabolic process (GO: 0006641), ribosomal subunit (GO: 0044391), cytoplasmic part (GO: 0044444) and mitochondrial envelope (GO: 0005740). The most significantly enriched KEGG pathways included pathways in oxidative phosphorylation (aga00190), proteasome (aga03050), ribosome (aga03010), metabolic pathways (aga01100) and endocytosis (aga04144). asimiR-nov9 and asi-miR-nov8 were identified to target oxidative phosphorylation and metabolic pathway, and miR-nov5 was also found to target proteasome and endocytosis. In addition, miRNA:mRNA interaction was analyzed through network generation as shown in Additional file 6: Figure S1. We identified 105 transcripts that were targeted by two or more than two miRNAs. Fifty transcripts (such as AGAP000951, AGA P003493, AGAP003720, AGAP004400, AGAP004880 and AGAP008327) were targeted by miRNAs in different transcript regions. Some of these are of great importance during the developmental signaling pathway and immune pathway. For example, AGAP003493 is one member of sugar transporter which facilitates the transport across cytoplasmic or internal membranes of a variety of substrates [37]. Also, AGAP008327 has a significant differential expression in the female salivary gland and 1.4-fold up-regulation with respect to highintensity Plasmodium berghei infection [38].

\section{Conserved miRNA in An. sinensis and its functions}

Previous evidence showed that miRNAs could negatively regulate the immune response in An. gambiae [18, 39]. Similarly, we identified the published conserved orthologues of miRNAs and multiple corresponding target transcripts of the immune pathway in An. gambiae. In total, we identified 21 conserved miRNAs and 8 mRNA counterparts in An. sinensis (Additional file 7: Figure S2). PGRP (peptidoglycan recognition protein), Caspar, and Rel2 (NF- $K B$ transcription factor) have been reported to play crucial roles in the immune signaling pathway [40]. Further analysis by miRNA-mRNA interaction network showed that asi-miR-275 and asi-miR-305 contained predicted binding sites within the 3'UTR of PGRP-LB 


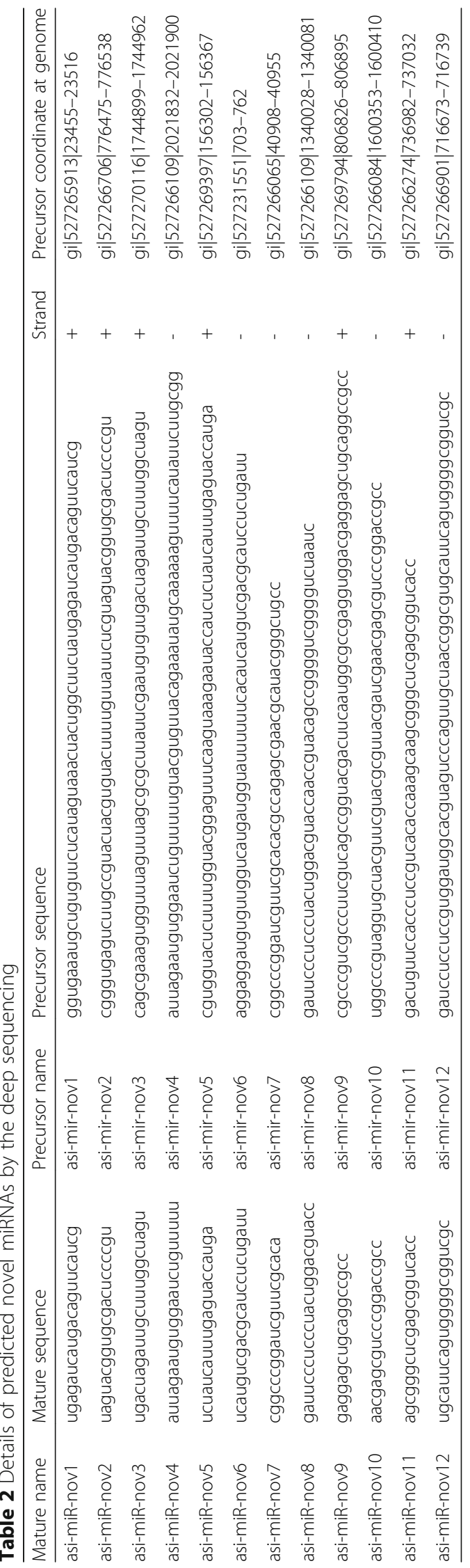




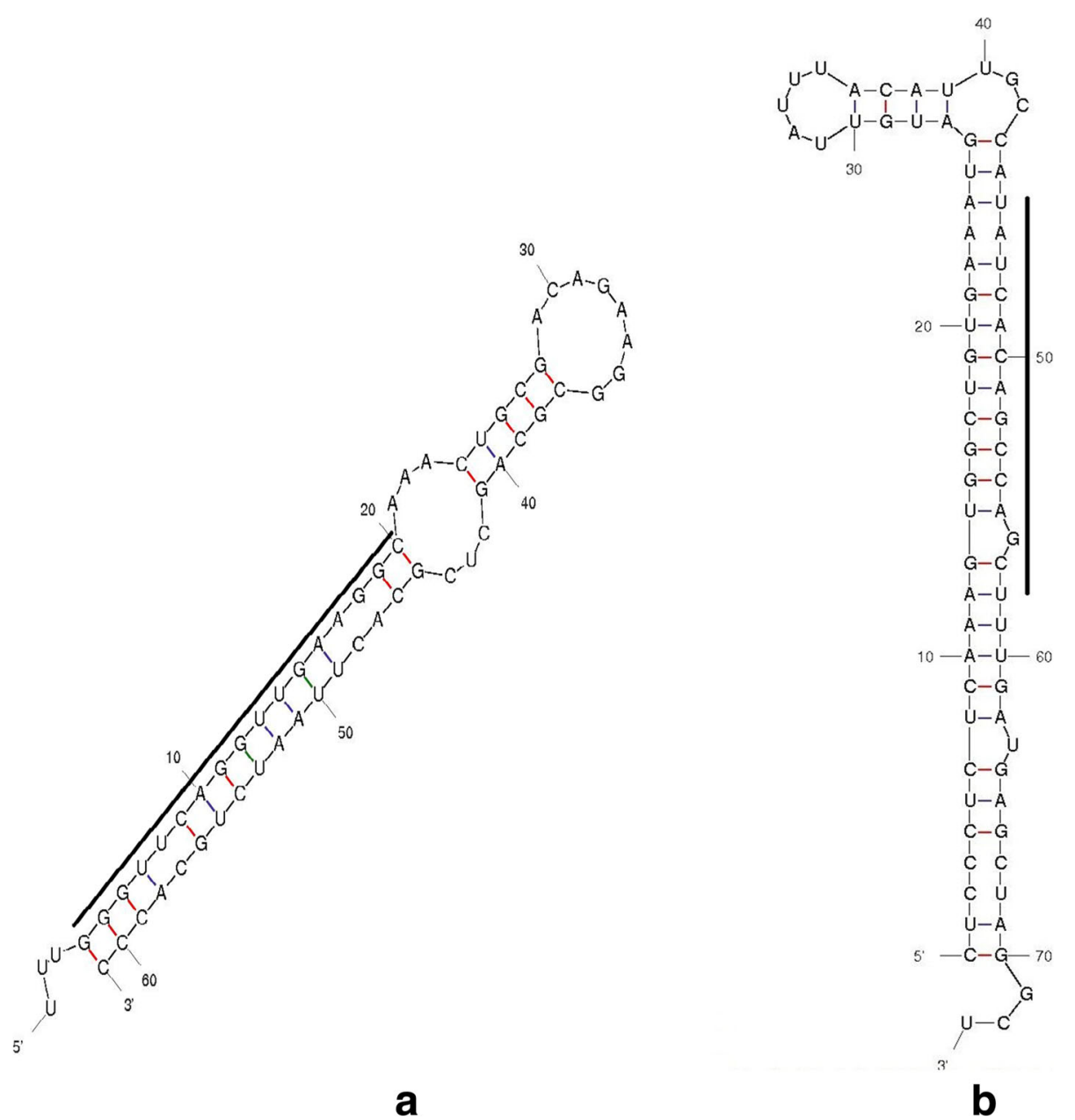

Fig. 2 Precursor miRNA hairpin structures of An. sinensis, the underlined nucleotides indicate the mature miRNAs. a Precursor miRNA hairpin structures of asi-mir-9383. b Precursor miRNA hairpin structures of asi-mir-2a-2

and $A P L 1 C$, respectively, and the 3'-UTR of the antiPlasmodium genes, such as PGRP-LC, PGRP-LD, Rel2, Caspar, IMD (immune deficiency pathway) and LRRD7/APL2, had in silico-predicted binding sites of many miRNAs. For instance, $P G R P-L D$ had the potential to interact with asi-miR-100, asi-miR-124, asimiR-210, asi-miR-278, asi-miR-282, and asi-miR-989. The asi-mir-2 was found to interact with the three key immune genes in IMD pathway that lead to an increased expression of antimicrobial peptides [18]. These results indicate that interaction of miRNAs with their targets could lead to regulation of immune proteins which further mediate midgut infection in An. sinensis.

\section{Discussion}

miRNAs have been discovered in many mosquito species, including An. gambiae, Ae. aegypti and Cx. fatigans $[14,16-18,41]$. In the present study, the miRNA expression profile of An. sinensis was analyzed by NGS technology and compared with the predicted results of the bioinformatic approach. From the An. sinensis small RNA data set, we identified a total of 49 conserved miRNAs which belong to 46 miRNA families, and 12 non-conserved (novel)

Table 3 Details of predicted new miRNAs by the bioinformatic approach

\begin{tabular}{|c|c|c|c|c|c|c|}
\hline Name & $\begin{array}{l}\text { Source } \\
\text { miRNA }\end{array}$ & Source organism & Mature sequence & Strand & $\begin{array}{l}\text { Minimum free energy values } \\
\text { (kcal/mol) }\end{array}$ & Coordinates in genome \\
\hline $\begin{array}{l}\text { asi-miR- } \\
9383\end{array}$ & $\begin{array}{l}\text { dme-miR- } \\
9383\end{array}$ & $\begin{array}{l}\text { Drosophila } \\
\text { melanogaster }\end{array}$ & GGGUUCAGGUUGAAGGCAAACU & $5^{\prime}$ & -24.2 & KE525292.1:9200-9260 \\
\hline $\begin{array}{l}\text { asi-miR- } \\
2 a-2\end{array}$ & $\begin{array}{l}\text { dme-miR- } \\
2 a-2\end{array}$ & $\begin{array}{l}\text { Drosophila } \\
\text { melanogaster }\end{array}$ & UAUCACAGCCAGCUUUGAUGAGC & $5^{\prime}$ & -29.5 & $\begin{array}{l}\text { KE525350.1:1783756- } \\
1783828\end{array}$ \\
\hline
\end{tabular}




\section{a}

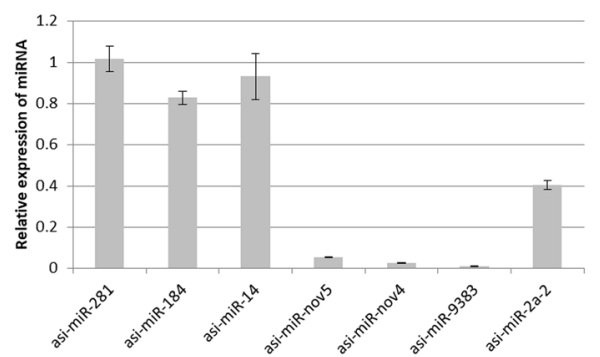

b

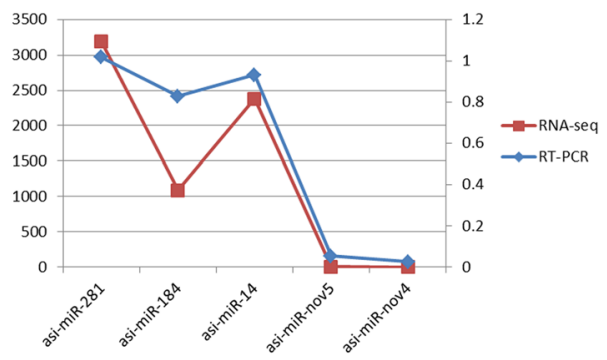

Fig. 3 Validation of the selected known and novel miRNAs by quantitative real-time PCR. a Seven miRNAs (five miRNAs from the sequencing result and two miRNAs from the bioinformatic approach) were identified by qRT-PCR. $\mathbf{b}$ The relative expression of five miRNAs from the sequencing result showed similar expression pattern when confirmed by qRT-PCR. The transcript levels of both known and novel miRNAs were calculated relative to the amount of U6 small nuclear after normalization. The real time PCR data with bars represent the mean \pm SD from three independent experiments

miRNAs. This indicates that the number of conserved miRNAs is probably much larger than that of novel miRNAs in An. sinensis. In addition, after a careful evaluation of the secondary structure analysis, another 42 conserved and two novel miRNAs were yielded by the bioinformatic approach for orthologous signatures in 26 organisms of the subphylum Hexapoda. In total, 14 miRNAs were assigned as the novel in this study. Among them, 12 miRNAs lack orthologues with recorded species in Vectorbase, indicating that these miRNAs could be unique for An. sinensis.

The number of miRNAs determined through our pipeline is almost consistent with what has been predicted

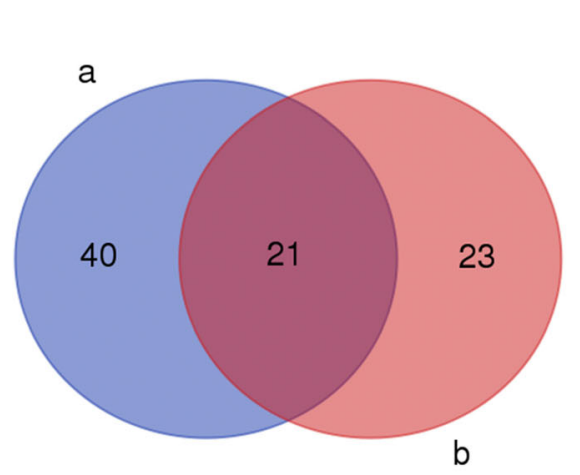

a
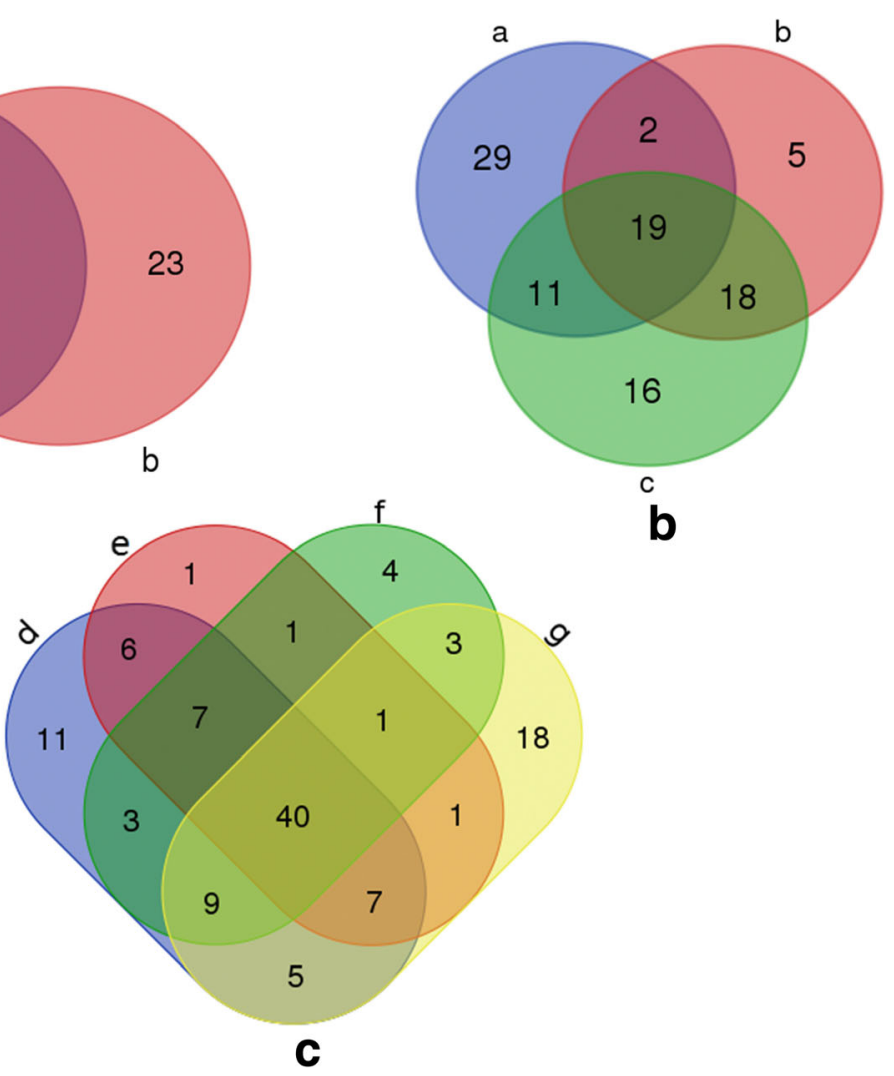

b

Fig 4 a Venn diagrams of the number of miRNAs predicted by deep sequencing and bioinformatic approach in An. sinensis (a, miRNAs predicted by deep sequencing; $b$, miRNAs predicted by bioinformatic approach). $\mathbf{b}$ Venn diagrams of the number of miRNAs predicted by deep sequencing, bioinformatic approach and miRNAs genes from genome in An. sinensis (a, miRNAs predicted by deep sequencing; $b$, miRNAs predicted by bioinformatic approach; c, miRNAs from genome prediction). c Venn diagrams of miRNAs for Ae. aegypti, An. gambiae, Cx. quinquefasciatus and An. sinensis from this study ( $d$, mature miRNAs of Ae. aegypti; e, mature miRNAs of An. gambiae; $f$, mature miRNAs of $C x$. quinquefasciatus; g, mature miRNAs of An. sinensis from this study) 


\section{GO Terms}

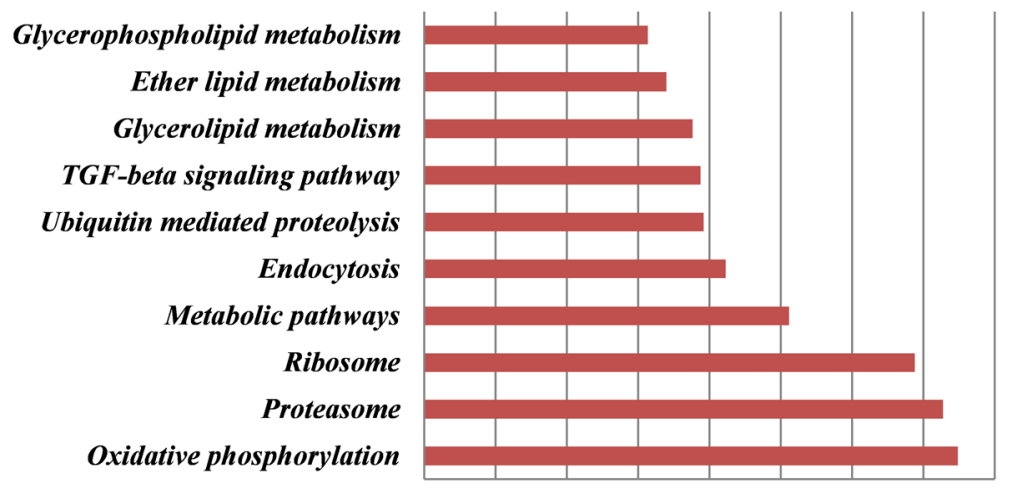

KEGG Pathways

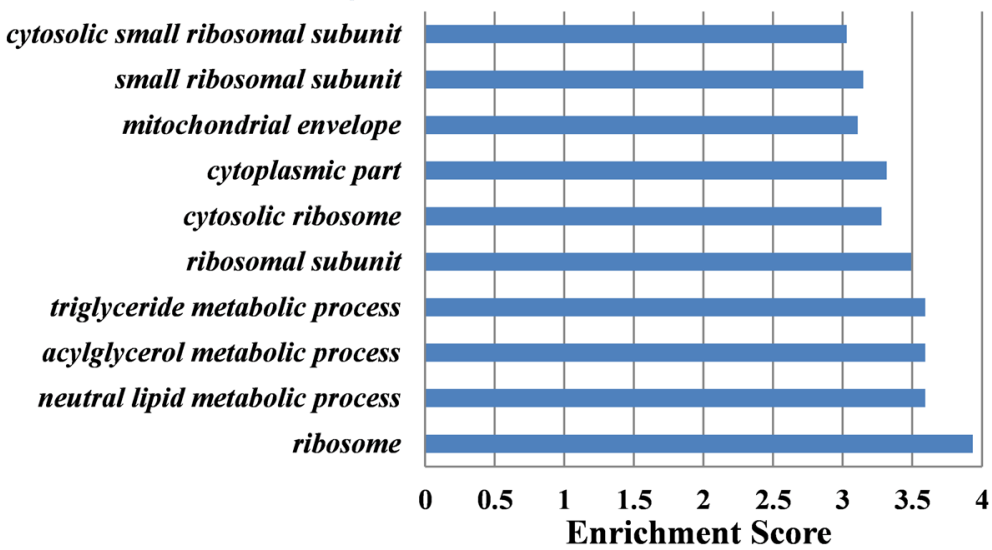

Fig. 5 KOBAS analysis of miRNA targets predicted by RNA hybrid. Bar charts represent top 10 Gene Ontology terms and KEGG pathways targeted by miRNAs. Enrichment score was calculated by $-\log 10(P<0.05)$

earlier for other mosquito species, such as 65 miRNAs for An. gambiae, 124 miRNAs for Ae. aegypti, 93 miRNAs for Cx. quinquefasciatus [42]. More interestingly, 40 miRNAs were found to be shared by all three taxa analyzed above. Of those, some conserved miRNAs have been found in a large variety of insect species, which indicate a highly conserved structure or role in evolution. In our study, the most abundantly expressed miRNAs, such as miR-8, miR-10, miR-184 and miR-281, exhibit a comparable expression pattern in An. gambiae [43]. In addition, some miRNAs, for instance, miR-184 was also reported as the most frequently occurring miRNA in other mosquito species $[15,44]$. In brief, comparisons between miRNA profiles by two approaches revealed the fundamental miRNA profile in Chinese malaria vector An. sinensis. However, the temporal and spatial expression patterns and related functions, still need to be further validated through biological experiments.

To validate our sequencing and the bioinformatic approach results, we performed qRT-PCR analysis using the RNA sample used for small RNA NGS. Eight miRNAs from our sequencing results and novel miRNAs from the bioinformatic approach were selected for validation. All selected miRNAs (asi-miR-281, asi-miR-184, asi-miR-14, asi-miR-nov5, asi-miR-nov4, asi-miR-9383 and asi-miR-2a) showed in the sequencing sample. Five miRNAs showed similar expression patterns as those revealed by our sequencing analysis, which indicated a low false discovery rate of our sequencing data and supported the validity of the profiling. Two selected miRNAs (asi-miR-281, asi-miR-184, asi-miR-14, asi-miR-nov5, asimiR-nov4, asi-miR-9383 and asi-miR-2a) from the bioinformatic approach result could also be confirmed by quantitative real-time PCR, indicating that we may have omitted some novel miRNA prediction during analyzing sequencing results.

The majority of known mosquito miRNAs have been determined through traditional direct cloning [45], bioinformatic prediction $[16,28]$ or deep sequencing $[15,46]$. In the present study, both bioinformatic prediction and the deep sequencing were applied. Next-generation sequencing (NGS) has become an innovative tool with the unprecedented depth of coverage to uncover miRNAs in many species [47]. This new approach has the ability to 
find both old and new miRNAs profiles by high throughput and fine resolution at a single base. However, sequences with low abundance may not be detected and predictive ability may be restricted if there is no reference genome [48]. Besides, the bioinformatic prediction is also widely used for the identification of miRNAs depending on similarity searches or algorithms based on evolutionary conservation [49]. However, it may miss species-specific and rapidly evolved miRNAs. In addition, miRNAs have significant variation in their expression levels in different time scales and tissues or under different conditions. This also makes it difficult to identify miRNAs by a single method [8]. Thus, as an alternative, joint usage of NGS and bioinformatics technology by taking advantage of their own strengths would undoubtedly facilitate the discrimination of vectors' miRNA profiles.

To understand various biological processes of miRNAs, it is necessary to study their potential targets. A total of 938 potential targets were predicted for the 14 novel miRNAs. All predicted targets have crucial biological roles ranging from metabolic process to mitochondrial envelope which might be important for development. Some KEGG pathways related to oxidative phosphorylation, proteasome and ribosome were also identified. More than one hundred transcripts were targeted by two or more miRNAs. Meanwhile, 50 transcripts were regulated by miRNAs in different regions, suggesting that miRNAs are involved in complicated regulatory networks as suggested by other studies $[8,36,50]$. The conserved miRNAs in An. sinensis were found to have in silico-predicted binding sites within many transcripts as described before in An. gambiae [18]. Significantly, these conserved orthologues play important roles in regulating pathogen infection [39]. Although, miRNA target genes are commonly recognized and bound by the miRNA seed region at the 3'-UTR, the recently identified miRNA binding sites within the 5'-UTR as well as the coding regions of target genes yield more target genes for interest miRNAs [9]. Deciphering the function of these cleaved targets identified in $A n$. sinensis would provide us with a better idea about their role in both mosquito biology and pathogen infection.

\section{Conclusions}

In conclusion, our study provides a comprehensive account of the miRNA profile of An. sinensis by combining the deep sequencing and the bioinformatic approach. Efforts were undertaken to understand the targets of miRNAs which can provide a better understanding of their biological function in mosquito biology and immunity and provide implications for effective control in the future. However, further experimental studies will be needed to validate these predictions and their interactions.

\section{Additional files}

Additional file 1: Table S1. miRNA-specific primers used in $\mathrm{QRT}$-PCR validation. (XLSX $10 \mathrm{~kb}$ )

Additional file 2: Table S2. miRNAs predicted from small RNA sequencing data of An. sinensis. (XLSX $33 \mathrm{~kb}$ )

Additional file 3: Table S3. miRNAs predicted by the bioinformatic approach. (XLSX $141 \mathrm{~kb}$ )

Additional file 4: Table S4. miRNA genes predicted from the genome of An. sinensis. (XLSX $17 \mathrm{~kb}$ )

Additional file 5: Table S5. List of miRNA targets identified by RNAhybrid on An. sinensis genes. (XLSX 35 kb)

Additional file 6: Figure S1. miRNA:mRNA interaction network of novel miRNAs and their predicted targets. Interaction network of novel miRNAs and their targets. miRNAs are diamond-shaped whereas targets are elliptic. Transcripts targeted by two or more than two miRNAs are marked in yellow color. (PDF $64 \mathrm{~kb}$ )

Additional file 7: Figure S2. miRNA:mRNA interaction network of conserved miRNAs and their predicted targets in the immune pathway. (TIFF $897 \mathrm{~kb}$ )

\section{Abbreviations}

GO: Gene ontology; IMD: Immune deficiency pathway; KEGG: Kyoto Encyclopedia of Genes and Genomes; miRNAs: microRNAs; NGS: Nextgeneration sequencing; PGRP: Peptidoglycan recognition protein; qRTPCR: quantitative reverse transcription real-time polymerase chain reaction; sRNAs: small RNAs; UTR: Untranslated regions

\section{Acknowledgments}

The authors would like to thank Ms Dongxiao Yang from the School of Bioinformatics, Nanjing Agricultural University for providing the bioinformatic algorithm involved in the work.

\section{Funding}

This work was supported by the National Natural Science Foundation of China (Nos. 81271867 and 91431104), International Science and Technology Cooperation Program of China (No. 2014DFA31130) and National Science \& Technology Major Program (No. 2009ZX10004-302). This work was also supported by Shanghai Municipal Commission of Health and Family Planning Grant (No. 20174Y0089).

\section{Availability of data and materials}

The datasets supporting the conclusions of this article are available in the Gene Expression Omnibus repository, (GSE109005, https:// www.ncbi.n/m.nih.gov/geo/query/acc.cgi?acc=GSE109005). miRNA-specific primers used in qRT-PCR validation available in Additional file 1: Table S1. miRNAs predicted from small RNA sequencing data of An. sinensis are available in Additional file 2: Table S2. miRNAs predicted by the bioinformatic approach are available in Additional file 3: Table S3. miRNA genes predicted from the genome of An. sinensis are in Additional file 4: Table S4. miRNA targets identified by RNAhybrid on An. sinensis genes are in Additional file 5: Table S5.

\section{Authors' contributions}

XYF and WH conceived and designed the study. XYF and XJZ analyzed the data and drafted the manuscript. JWW and SSZ critically revised the manuscript. All authors read and approved the final manuscript.

Ethics approval and consent to participate

Not applicable.

Consent for publication

Not applicable.

Competing interests

The authors declare that they have no competing interests. 


\section{Publisher's Note}

Springer Nature remains neutral with regard to jurisdictional claims in published maps and institutional affiliations.

\begin{abstract}
Author details
'National Institute of Parasitic Diseases, Chinese Center for Disease Control and Prevention, Key Laboratory of Parasite and Vector Biology, National Health and Family Planning Commission, WHO Collaborating Center for Tropical Diseases, National Center for International Research on Tropical Diseases, Shanghai 200025, People's Republic of China. ${ }^{2}$ Joint Research Laboratory of Genetics and Ecology on Parasites-hosts Interaction, National Institute of Parasitic Diseases - Fudan University, Shanghai 200025, China. ${ }^{3}$ Institute of Software Engineering, Zhejiang University, Hangzhou 310011, China. ${ }^{4}$ State Key Laboratory of Genetic Engineering, Ministry of Education Key Laboratory of Contemporary Anthropology, Collaborative Innovation Center for Genetics and Development, School of Life Sciences, Fudan University, Shanghai 200438, People's Republic of China.
\end{abstract}

Received: 14 December 2017 Accepted: 20 February 2018

Published online: 12 March 2018

\section{References}

1. World Health Organization. World Malaria Report. Geneva: WHO; 2014.

2. Zhang L, Zhou SS, Feng J, Fang W, Xia ZG. [Malaria situation in the People's Republic of China in 2014]. [Zhongguo ji sheng chong xue yu ji sheng chong bing za zhi] Chin J Parasit Parasitic Dis. 2015:33(5):319-26. (In Chinese).

3. Zhou S, Li Z, Cotter C, Zheng C, Zhang Q, Li H, et al. Trends of imported malaria in China 2010-2014: analysis of surveillance data. Malar J. 2016;15(1):39.

4. Ren Z, Wang D, Ma A, Hwang J, Bennett A, Sturrock HJ, et al. Predicting malaria vector distribution under climate change scenarios in China: challenges for malaria elimination. Sci Rep. 2016;6

5. Zhou X-N, Xia Z-G, Wang R-B, Qian Y-J, Zhou S-S, Utzinger J, et al. Feasibility and roadmap analysis for malaria elimination in China. Adv Parasitol. 2014; 86:21-46.

6. Bai L, Morton LC, Liu Q. Climate change and mosquito-borne diseases in China: a review. Glob Health. 2013:9(1):1.

7. Chang X, Zhong D, Fang Q, Hartsel J, Zhou G, Shi L, et al. Multiple resistances and complex mechanisms of Anopheles sinensis mosquito: a major obstacle to mosquito-borne diseases control and elimination in China. PLoS Negl Trop Dis. 2014;8(5):e2889.

8. Asgari S. Role of microRNAs in arbovirus/vector interactions. Viruses. 2014 6(9):3514-34.

9. Guil S, Esteller M. RNA-RNA interactions in gene regulation: the coding and noncoding players. Trends Biochem Sci. 2015;40(5):248-56.

10. Behura SK. Insect microRNAs: Structure, function and evolution. Insect Biochem Mol Biol. 2007;37(1):3-9.

11. Wightman $\mathrm{B}, \mathrm{Ha} \mathrm{I}$, Ruvkun $\mathrm{G}$. Posttranscriptional regulation of the heterochronic gene lin-14 by lin-4 mediates temporal pattern formation in C. elegans. Cell. 1993;75(5):855-62.

12. Lee RC, Feinbaum RL, Ambros V. The C. elegans heterochronic gene lin-4 encodes small RNAs with antisense complementarity to lin-14. Cell. 1993; 75(5):843-54.

13. Liu W, Huang H, Xing C, Li C, Tan F, Liang S. Identification and characterization of the expression profile of microRNAs in Anopheles anthropophagus. Parasit Vectors. 2014;7:159.

14. Hong S, Guo Q, Wang W, Hu S, Fang F, LV Y, et al. Identification of differentially expressed microRNAs in Culex pipiens and their potential roles in pyrethroid resistance. Insect Biochem Mol Biol. 2014:55C:39-50.

15. Skalsky RL, Vanlandingham DL, Scholle F, Higgs S, Cullen BR. Identification of microRNAs expressed in two mosquito vectors, Aedes albopictus and Culex quinquefasciatus. BMC Genomics. 2010;11:119.

16. Chatterjee R, Chaudhuri K. An approach for the identification of microRNA with an application to Anopheles gambiae. Acta Biochim Pol. 2006;53(2): 303-9.

17. Bryant B, Macdonald W, Raikhel AS. microRNA miR-275 is indispensable for blood digestion and egg development in the mosquito Aedes aegypti. Proc Natl Acad Sci USA. 2010;107(52):22391-8.

18. Dennison NJ, BenMarzouk-Hidalgo OJ, Dimopoulos G. MicroRNA-regulation of Anopheles gambiae immunity to Plasmodium falciparum infection and midgut microbiota. Dev Comp Immunol. 2015;49(1):170-8.
19. Jain S, Rana V, Shrinet J, Sharma A, Tridibes A, Sunil S, et al. Blood feeding and Plasmodium infection alters the miRNome of Anopheles stephensi. PloS One. 2014;9(5):e98402.

20. Liu Y, Zhou Y, Wu J, Zheng P, Li Y, Zheng X, et al. The expression profile of Aedes albopictus miRNAs is altered by dengue virus serotype-2 infection. Cell Biosci. 2015:5:16

21. Li R, Li Y, Kristiansen K, Wang J. SOAP: short oligonucleotide alignment program. Bioinformatics. 2008;24(5):713-4.

22. Kozomara A, Griffiths-Jones S. miRBase: annotating high confidence microRNAs using deep sequencing data. Nucleic Acids Res. 2014;42(D1): D68-73.

23. Griffiths-Jones S, Bateman A, Marshall M, Khanna A, Eddy SR. Rfam: an RNA family database. Nucleic Acids Res. 2003;31(1):439-41.

24. Gelfand Y, Rodriguez A, Benson G. TRDB -The Tandem Repeats Database. Nucleic Acids Res. 2007:35:D80-D7.

25. An J, Lai J, Lehman ML, Nelson CC. MiRDeep*: An integrated application tool for miRNA identification from RNA sequencing data. Nucleic Acids Res. 2012;41(2):727-37

26. Allen E, Xie Z, Gustafson AM, Carrington JC. microRNA-directed phasing during trans-acting siRNA biogenesis in plants. Cell. 2005;121(2):207-21.

27. Friedlander MR, Chen W, Adamidi C, Maaskola J, Einspanier R, Knespel S, et al. Discovering microRNAs from deep sequencing data using miRDeep. Nat Biotechnol. 2008:26(4):407-15.

28. Krishnan R, Kumar V, Ananth V, Singh S, Nair AS, Dhar PK. Computational identification of novel microRNAs and their targets in the malarial vector, Anopheles stephensi. Syst Synth Biol. 2015;9(1-2):11-7.

29. Zuker M. Mfold web server for nucleic acid folding and hybridization prediction. Nucleic Acids Res. 2003;31(13):3406-15.

30. Xue C, Li F, He T, Liu GP, Li Y, Zhang X. Classification of real and pseudo microRNA precursors using local structure-sequence features and support vector machine. BMC Bioinformatics. 2005;6:310.

31. Lei Z, Lv Y, Wang W, Guo Q, Zou F, Hu S, et al. MiR-278-3p regulates pyrethroid resistance in Culex pipiens pallens. Parasitol Res. 2015;114(2) 699-706.

32. Kruger J, Rehmsmeier M. RNAhybrid: microRNA target prediction easy, fast and flexible. Nucleic Acids Res. 2006:34:W451-4.

33. Grillo G, Turi A, Licciulli F, Mignone F, Liuni S, Banfi S, et al. UTRdb and UTRsite (RELEASE 2010): a collection of sequences and regulatory motifs of the untranslated regions of eukaryotic mRNAs. Nucleic Acids Res. 2010;38: D75-80.

34. Xie C, Mao X, Huang J, Ding Y, Wu J, Dong S, et al. KOBAS 2.0: a web server for annotation and identification of enriched pathways and diseases. Nucleic Acids Res. 2011;39:W316-22.

35. Shannon P, Markiel A, Ozier O, Baliga NS, Wang JT, Ramage D, et al. Cytoscape: a software environment for integrated models of biomolecular interaction networks. Genome Res. 2003;13(11):2498-504.

36. Dritsou V, Deligianni E, Dialynas E, Allen J, Poulakakis N, Louis C, et al. Noncoding RNA gene families in the genomes of anopheline mosquitoes. BMC Genomics. 2014;15:1038.

37. Holt RA, Subramanian GM, Halpern A, Sutton GG, Charlab R, Nusskern DR, et al. The genome sequence of the malaria mosquito Anopheles gambiae. Science. 2002;298(5591):129-49.

38. Mendes AM, Awono-Ambene PH, Nsango SE, Cohuet A, Fontenille D, Kafatos FC, et al. Infection intensity-dependent responses of Anopheles gambiae to the African malaria parasite Plasmodium falciparum. Infect Immun. 2011;79(11):4708-15.

39. Winter F, Edaye S, Huttenhofer A, Brunel C. Anopheles gambiae miRNAs as actors of defence reaction against Plasmodium invasion. Nucleic Acids Res. 2007;35(20):6953-62.

40. Cirimotich CM, Dong Y, Garver LS, Sim S, Dimopoulos G. Mosquito immune defenses against Plasmodium infection. Dev Comp Immunol. 2010;34(4): 387-95.

41. Liu S, Lucas KJ, Roy S, Ha J, Raikhel AS. Mosquito-specific microRNA-1174 targets serine hydroxymethyltransferase to control key functions in the gut. Proc Natl Acad Sci USA. 2014;111(40):14460-5.

42. Griffiths-Jones S, Grocock RJ, van Dongen S, Bateman A, Enright AJ. miRBase: microRNA sequences, targets and gene nomenclature. Nucleic Acids Res. 2006:34:D140-4.

43. Biryukova I, Ye T, Levashina E. Transcriptome-wide analysis of microRNA expression in the malaria mosquito Anopheles gambiae. BMC Genomics. 2014;15:557 
44. Li S, Mead EA, Liang S, Tu Z. Direct sequencing and expression analysis of a large number of miRNAs in Aedes aegypti and a multi-species survey of novel mosquito miRNAs. BMC Genomics. 2009;10:581.

45. Mead EA, Tu Z. Cloning, characterization, and expression of microRNAs from the Asian malaria mosquito, Anopheles stephensi. BMC Genomics. 2008;9:244.

46. Shrinet J, Jain S, Jain J, Bhatnagar RK, Sunil S. Next generation sequencing reveals regulation of distinct Aedes microRNAs during chikungunya virus development. PLoS Negl Trop Dis. 2014;8(1):e2616.

47. Yoon S, De Micheli G. Computational identification of microRNAs and their targets. Birth Defects Res C Embryo Today. 2006;78(2):118-28.

48. Jain S, Rana V, Tridibes A, Sunil S, Bhatnagar RK. Dynamic expression of miRNAs across immature and adult stages of the malaria mosquito Anopheles stephensi. Parasit Vectors. 2015:8:179.

49. Li L, Xu J, Yang D, Tan X, Wang H. Bioinformatic approaches for microRNA studies: a review. Mamm Genome. 2010;21(1-2):1-12.

50. Lucas KJ, Myles KM, Raikhel AS. Small RNAs: a new frontier in mosquito biology. Trends Parasitol. 2013;29(6):295.

Submit your next manuscript to BioMed Central and we will help you at every step:

- We accept pre-submission inquiries

- Our selector tool helps you to find the most relevant journal

- We provide round the clock customer support

- Convenient online submission

- Thorough peer review

- Inclusion in PubMed and all major indexing services

- Maximum visibility for your research

Submit your manuscript at www.biomedcentral.com/submit
C) Biomed Central 